\title{
Comparison of lateral controllers for autonomous vehicle : experimental results
}

\author{
Salvador DOMINGUEZ ${ }^{1}$, Alan ALI ${ }^{1}$, Gaëtan GARCIA ${ }^{1}$ and Philippe MARTINET $^{1}$
}

\begin{abstract}
A good path tracker is one of the keys for the successful development of a self-driving car. In the literature, there exists a wide variety of techniques, some complex and some simple and yet effective in particular scenarios. The choice of the path tracker influences the performance in terms of precision, stability and passenger comfort. This paper addresses the lateral control of a self-driving car in an urban environment, where speed is not high but variations in velocity and curvature are frequent. In choosing a lateral controller, simplicity, efficiency and robustness are considered as the main criteria. In this paper, three classical techniques used for controlling the lateral error are analyzed: pure pursuit, Stanley and a simplified kinematic steering control. Additionally, a novel kinematic controller based on the lateral speed is proposed. A home-made realistic simulation environment has been developed to allow rapid testing of the control laws. The relevance of this work has been demonstrated for all controllers through realistic simulations and experiments. The experimental site is the campus of Ecole Centrale de Nantes, where all control laws have been compared along the same path. A longer path, involving a portion of the ring road of Nantes (France) has been simulated. It involves speeds up to 90 $\mathrm{km} / \mathrm{h}$, allowing to extrapolate the comparison results to higher velocities.
\end{abstract}

\section{INTRODUCTION}

Nowadays, autonomous vehicle projects are among the most important ongoing research and development areas in robotics. Automated vehicles are useful to increase traffic density, efficiency, safety and comfort. Many intelligent vehicles and ITS solutions have already been implemented (e.g. Google Car, VIP), and car manufacturers have already developed solutions (Tesla, Audi, Mercedes, Renault, PSA, Nissan, Fiat...). Research and development efforts strive for improved performance, higher reliability and lower cost.

The control of automated vehicles involves coupled lateral and longitudinal dynamics. Decoupling these two dynamics is straightforward when using a kinematic vehicle model [20], however a kinematic model cannot be used in high velocity applications. In highway applications, where road curvature is low, it is common to assume that the two dynamics are decoupled, allowing independent design of the longitudinal and lateral control laws [14]. In urban areas, the authors of [7] have implemented lateral control assuming that it is sufficiently independent from longitudinal control. In [12], lateral and longitudinal controllers have been designed independently. The parameters of the lateral controller have been calculated for each speed, and saved in a lookup table. However, in case of sporty driving at high speed

\footnotetext{
${ }^{1}$ Institut de Recherche en Communications et Cybernétique de Nantes (IRCCyN), Ecole Centrale de Nantes (ECN), Nantes, France
}

and/or on tightly curved roads, the coupling between the two dynamics cannot be ignored, and decoupling the two dynamics becomes more difficult.

The objective of lateral control is to follow a desired path by making lateral and angular errors equal to zero. The path can be generated off-line (predefined path) or on-line (to avoid obstacles, to change lane...). Lateral control can also be used for lane keeping applications. The lane can be identified by integrating magnetic markers in the roads [15], which is usually very costly, by detecting road lines [2] using cameras, or by following an already recorded path.

Many controllers have been used for lateral control. Some of them require an accurate kinematic or dynamic vehicle model (e.g. $H_{\infty}$ [11]), others use rough vehicle models (e.g. sliding mode [1], [17] or adaptative controllers [5], [21]), and some don't use any vehicle model (pure pursuit [16], Stanley [16], fuzzy logic [18]...).

In [21], an adaptive PID controller was implemented on a real automated vehicle, with enhanced performance with respect to the classical PID controller. An extended kinematic model was proposed in [9] in order to take into account two sliding parameters in a novel kinematic model which requires the use of an observer. This approach has yielded good results for off-road robots evolving at low velocities, like in agricultural applications. When velocity increases, it is necessary to use adaptive and predictive control to adapt the vehicle's behavior to the evolution of sliding, and predict vehicle motion with respect to the desired path [10].

A comparison between $H_{\infty}$, adaptive, PID and fuzzy controllers has been done in [5]. It has been concluded that the adaptive controller always yields the smaller errors. The fuzzy controller showed a good performance, but there is no stability proof for fuzzy controllers. Another interesting and detailed comparison between many controllers was performed in [16]. The comparison covered many scenarios and several velocities, to show the strengths and weaknesses of each control law. Based on simulation results only, it has been concluded that there is no perfect control law which can be used in all situations and at all velocities.

This paper deals with the lateral control. The speed reference is given by a longitudinal controller. It accelerates and decelerates progressively, either to reach the maximum allowed speed, when the road is straight, or to bound the centripetal acceleration in curves. The second section of the paper presents the classical kinematic model (Ackermann/bicycle model) which can be linearized exactly. The third section focuses on the four controllers (Pure pursuit, Stanley, Sliding control and a novel lateral speed controller) 


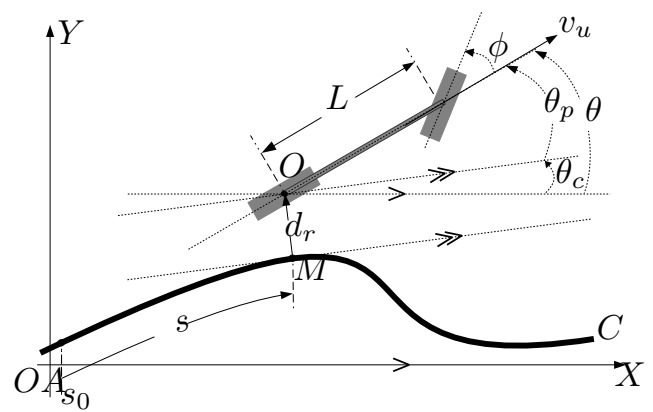

Fig. 1. Bicycle model

to be compared. After describing the experimental context, the last section shows the different controller behaviors on a pre-recorded path of about $1 \mathrm{~km}$ performed by the real car (on the campus of Ecole Centrale de Nantes, maximum allowable speed: $30 \mathrm{~km} / \mathrm{h}$ ), and a longer, simulated path where the velocity reaches $90 \mathrm{~km} / \mathrm{h}$.

\section{MODELING}

The classical kinematic model can only be used at low velocities, for comparatively light systems moving on sufficiently solid ground, typically asphalt. Heavy vehicles produce wheel deformation while turning, which in turn produces lateral slippage. Softer ground may also produce lateral slippage, in addition to longitudinal slippage. In this work, the popular Ackerman model [3] is used. The car is modeled as a bicycle (fig. 1), where $[O A, X, Y]$ is an absolute frame, $C$ is the reference path, $O$ is the center of the rear wheel of the vehicle, $M$ is the point of $C$ closest to $O, s$ is the curvilinear abscissa of point $M$ along $C, \theta_{c}(s)$ is the orientation of the tangent to $C$ at $M$ (with respect to the absolute frame), $\theta$ is the heading angle.

This model can be reformulated with respect to the reference path $C$ instead of the absolute frame:

$$
\left\{\begin{array}{l}
\dot{s}=\frac{\cos \theta_{p}}{1-c(s) d_{r}} v_{u} \\
\dot{d}_{r}=\sin \theta_{p} v_{u} \\
\dot{\theta}_{p}=\left(\frac{\tan \phi}{L}-c(s) \frac{\cos \theta_{p}}{1-c(s) d_{r}}\right) v_{u}
\end{array}\right.
$$

In system (1), $c(s)$ denotes the curvature of path $C$ at $M$, $\theta_{p}=\theta-\theta_{c}(s)$ is the angular deviation of the vehicle with respect to $C, d_{r}$ the rear lateral deviation of the vehicle with respect to $C, \phi$ is the steering angle (angle between the front wheel and the car body axis), $L$ is the vehicle wheelbase, and $v_{u}$ is the vehicle speed along its longitudinal axis. The inputs to this system are the steering angle $\phi$ and the speed of the car $v_{u}$. This model can be linearized using an exact linearization technique [6] by defining a new input $W_{1}$ to solve system (1) and then computing the steering angle by taking:

$$
\begin{gathered}
\phi=\operatorname{atan}\left(L\left(\frac{W_{1}}{v_{u}}+c(s) \frac{\cos \left(\theta_{p}\right)}{1-c(s) d_{r}}\right)\right) \\
v_{u} \neq 0
\end{gathered}
$$

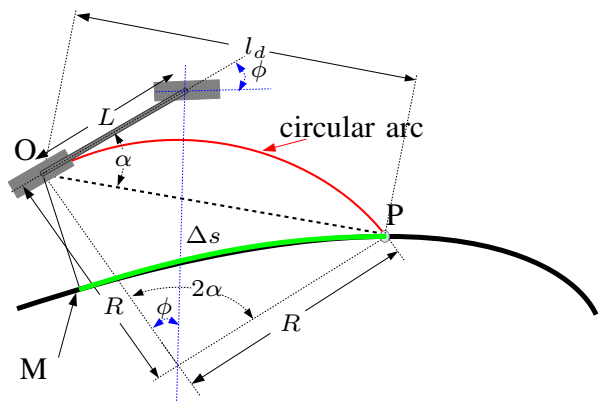

Fig. 2. Pure pursuit

Where $W_{1}$ is the new linearized input. This gives:

$$
\dot{\theta}_{p}=W_{1}
$$

So, a linear system can be obtained for the lateral motion, hence it can be easily controlled independently from the longitudinal controller.

\section{CONTROL}

In the following, three popular lateral control laws and our proposed lateral velocity controller are described. The first two laws are geometric controllers and don't require a kinematic model of the vehicle, whereas the last two use the kinematic model to compute the control output.

\section{A. Pure pursuit control law}

The pure pursuit is one of the most common lateral control strategies [16]. In this control law, a goal point is defined on the desired path, by taking a look ahead distance $l_{d}$ from the current position of the rear axle center $O$ to the desired path. Then the curvature of the arc that connects $O$ to the goal point is calculated geometrically (figure 2 ). It leads to calculating the required steering angle as follows:

$$
\frac{l_{d}}{\sin (2 \alpha)}=\frac{R}{\sin \left(\frac{\pi}{2}-\alpha\right)} \Longrightarrow \frac{l_{d}}{\sin (\alpha)}=2 R
$$

So:

$$
k_{\text {cur }}=\frac{1}{R}=\frac{2 \sin (\alpha)}{l_{d}}
$$

$k_{\text {cur }}$ is the curvature of the circular arc connecting the rear axle with the goal point.

From figure 2, it comes:

$$
\phi=\tan ^{-1}\left(k_{\text {cur }} L\right)
$$

From (5) and (6), the pure pursuit control law is defined as:

$$
\phi(t)=\tan ^{-1}\left(\frac{2 L \sin (\alpha(t))}{l_{d}}\right)
$$

Decreasing $l_{d}$ may make the system more accurate, but also more oscillatory. Conversely, increasing $l_{d}$ makes the tracking smoother but less accurate. It is usual to make $l_{d}$ proportional to velocity. In our case the pursuit point $P$ is defined as the point of the path whose curvilinear abscissa is ahead by $\Delta s$ (figure 2), with $\Delta s$ proportional to velocity. Then $l_{d}$ can be computed from $P$ and $O$. 


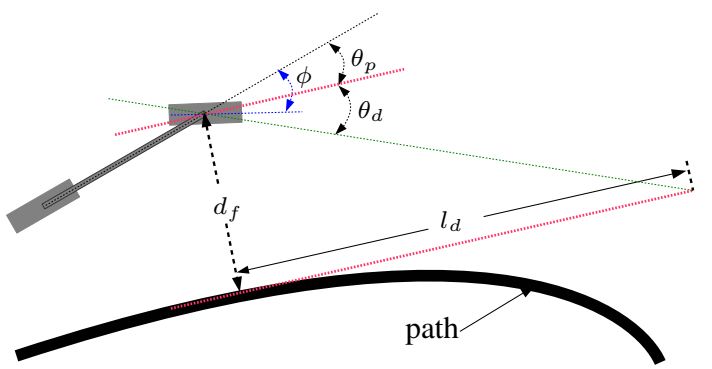

Fig. 3. Stanley control

\section{B. Stanley control law}

This control law was used in the autonomous vehicle of Stanford University which won the DARPA Grand Challenge in 2005. The Stanley control law is given by:

$$
\phi(t)=\theta_{p}(t)+\tan ^{-1}\left(\frac{d_{f}(t)}{l_{d}}\right)
$$

This control law consists of two terms. The first term is to compensate the angular error $\theta_{p}$ and the second term is to compensate the front lateral distance error $d_{f}$ measured form the centre of the front axle to the nearest point on the path. This error is also considered as angular error $\theta_{d}(t)=$ $\tan ^{-1}\left(\frac{d_{f}(t)}{l_{d}}\right)$. The parameter $l_{d}$ is a headway distance, which is usually chosen proportional to vehicle speed $l_{d}=\frac{v}{k_{v}}$ where $k_{v}$ is a constant.

$$
\phi(t)=\theta_{p}(t)+\tan ^{-1}\left(\frac{k_{v} d_{f}(t)}{v}\right)
$$

\section{Modified sliding mode control law}

The sliding mode control law is a simple and robust control law which does not require a precise model of the system and can also ensure stability even if the parameters of the system change slowly over time. A sliding surface $\psi$ can be defined as follows:

$$
\psi=k_{\theta p} \theta_{p}+k_{d} d_{r}
$$

where $k_{\theta p}, k_{d}$ are weighting coefficients. The sliding mode controller implies that:

$$
\dot{\psi}=-K_{\psi} \operatorname{sign}(\psi)
$$

The main disadvantage of the sliding control law is chattering, which can be reduced by choosing a higher order sliding mode controller [4]. The following controller ensures stability without chattering [1]:

$$
\dot{\psi}=-K_{\psi} \psi
$$

with $K_{\psi}$ a positive constant.

So from equations (12), (10) and (3), the following control is obtained:

$$
W_{1}=-\frac{\left(K_{\psi} k_{\theta p} \theta_{p}+K_{\psi} k_{d} d_{r}+k_{d} \dot{d}_{r}\right)}{k_{\theta p}}=\dot{\theta_{p}}
$$

which can be placed in equation (2) to obtain the reference steering angle. Note that $v_{u}$ must not be 0 in equation (2), so in practice we set a lower bound for the absolute value of $v_{u}$.

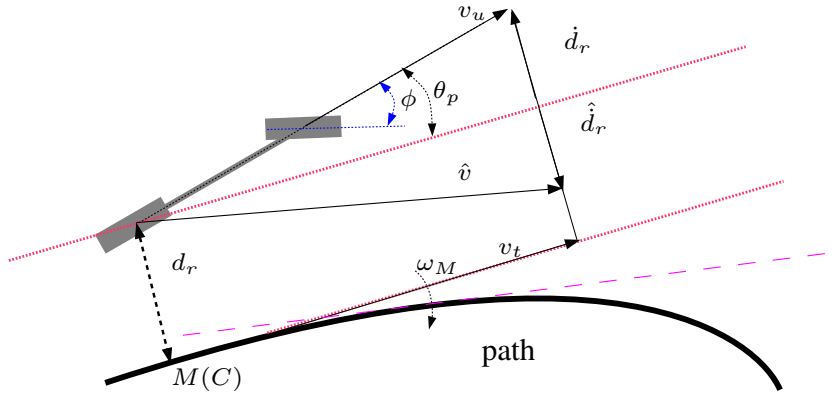

Fig. 4. Lateral speed control

\section{Kinematic lateral speed control law}

A new kinematic control law has been designed to control the rear lateral distance and the orientation error by controlling the lateral speed $\dot{d}_{r}$, which in turn is controlled by the angular speed of the car $\dot{\theta}$ through the steering angle $\phi$. The aim is to obtain a reasonable precision compared with other methods without sacrificing passenger comfort, and with good stability for speed ranges of interest (up to $90 \mathrm{~km} / \mathrm{h}$ ). As the lateral speed $\dot{d}_{r}$ is under control, the motions toward the path are smoother, after tuning the parameters properly.

Let us define the desired lateral speed $\hat{d}_{r}$ as the speed at which the rear axle must approach the line tangent to the path at $M, M$ being the point of the path closest to the rear axle center. If the car is far from that line, it is required to make it get closer at higher speed than if it is near, so the desired lateral speed $\hat{\dot{d}}_{r}$ can be defined as proportional to the lateral error $d_{r}$, with negative sign.

$$
\hat{\dot{d}}_{r}=-k_{l a t} d_{r}
$$

In practice, the absolute value of the desired lateral speed is limited to a reasonable maximum value, which in our case is $1 \mathrm{~m} / \mathrm{s}$. On the other hand, according to equation 1 the derivative of the lateral speed of the rear axle $\dot{d}_{r}$ is:

$$
\dot{d}_{r}=v_{u} \sin \left(\theta_{p}\right)
$$

Thus the lateral speed error $\dot{d}_{e r r}=\dot{d}_{r}-\hat{\dot{d}}_{r}$ writes:

$$
\dot{d}_{e r r}=v_{u} \sin \left(\theta_{p}\right)+k_{l a t} d_{r}
$$

In order to reduce the lateral speed error $\dot{d}_{e r r}$, the controller must steer the car in the direction of vector $\hat{v}$ of fig. 4 . The control variable of eq. (2) is defined as proportional to the lateral speed error $\dot{d}_{e r r}$, introducing the control gain $K_{\theta}$ with negative sign:

$$
W 1=-K_{\theta}\left(v_{u} \sin \left(\theta_{p}\right)+k_{l a t} d_{r}\right)
$$

So equation (2) becomes:

$$
\phi=\operatorname{atan}\left(L *\left(-K_{\theta} \sin \left(\theta_{p}\right)-\frac{K_{\theta} k_{l a t} d_{r}}{v_{u}}+\frac{c(s) \cos \left(\theta_{p}\right)}{1-c(s) d_{r}}\right)\right)
$$

As in the kinematic sliding mode control, the absolute value of speed $v_{u}$ must have a non zero lower bound. 


\section{EXPERIMENTAL SETUP}

The car used for the experiments is a Renault Zoe ZE converted to drive-by-wire and equipped with sensors which allow, among other things, to localize it in the surrounding environment with a precision of few centimeters, typically less than $5 \mathrm{~cm}$ [8]. The multi-map based localization method uses odometry and a laser scan as input. The laser scan is generated using the data provided by three planar SICK LMS151 LiDARs that measure in the same plane parallel to the floor at a height of $50 \mathrm{~cm}$ and covering the $360^{\circ}$ surrounding the car. This ensures a good localization even if some part of the environment is hidden. It also has a GPS receiver which is used mainly during the map building and path recording process and to initialize the position on the map in localization mode.

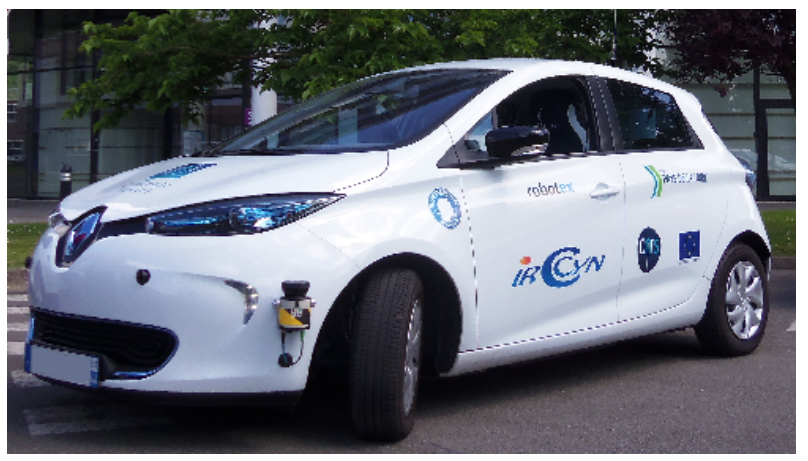

Fig. 5. Vehicle used for the experiments

A homemade realistic simulation environment has been developed under $\mathrm{C}++$ and ROS to allow fast prototyping of controllers. It is built on the same embedded software architecture as the one used in the real car. The only difference concerns the actuators of the vehicle which have been replaced by transfer functions whose parameters have been identified with the real car. The main difference between the simulator and the real car lies in the model of the contact with the ground.

A full path consists of a sequence of sub-map paths, which in turn are composed of a sequence of points. For path following, local path points are interpolated by a spline in order to have a continuous estimation of the position of the car with respect to the path, so the proposed path following controllers can be applied.

The aim of the first experiment is to check the results of the four controllers with the real car and also to compare them with the results in simulation. It has been performed on the campus of Ecole Centrale de Nantes (France), where the speed limit is $30 \mathrm{~km} / \mathrm{h}$. Figure 6 shows the $1 \mathrm{~km}$ long path.

The parameters of the controllers have been adjusted to obtain the best stable response in simulation and also with the real car as a result of intensive tests, meaning that different parameter settings would produce similar or worse results.

In order to check if common conclusions can be extracted with the real car and in simulation, the same experiment has

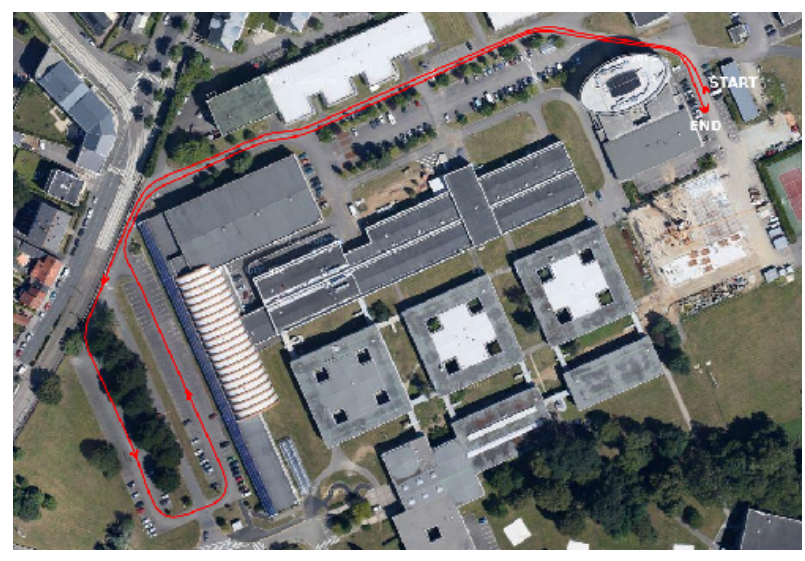

Fig. 6. Path used in the first experiment

also been performed in the simulation environment.

The second experiment is a $5 \mathrm{~km}$ long path for testing the controllers at higher speeds, up to $90 \mathrm{~km} / \mathrm{h}$. As the licence to drive the car in an autonomous mode on public roads is not yet available, the path has been recorded with the real car driven manually, and the controllers have been tested in the simulation environment along the recorded path.

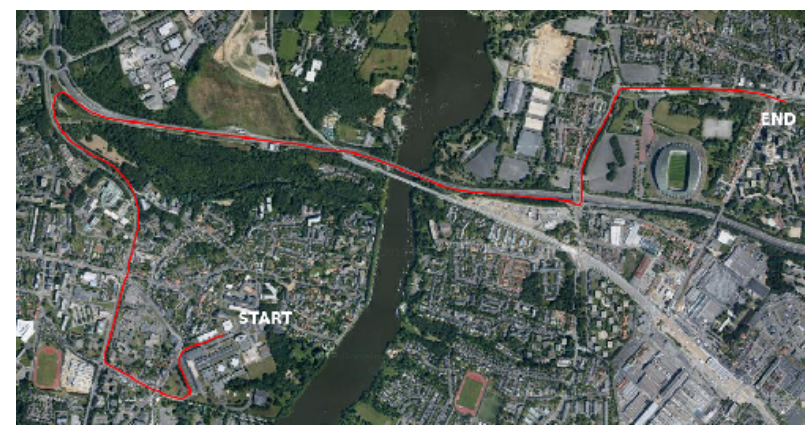

Fig. 7. Path used in the second experiment

\section{RESULTS}

Figure 8 shows the speed and curvature profiles along the first path. Figure 9 shows the lateral error obtained along the path for the four controllers.

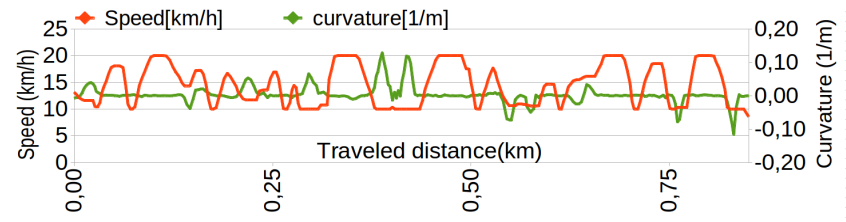

Fig. 8. Speed profile (in green) and curvature (in orange) vs. distance travelled in $\mathrm{km}$ in the first experiment.

The lateral errors obtained in simulation and with the real car for each of the four controllers are shown in figures 10 and 11. The whisker plots present the statistical distribution of the errors. As expected, the errors in simulation are lower than in the real case, due to not considering the irregularities 


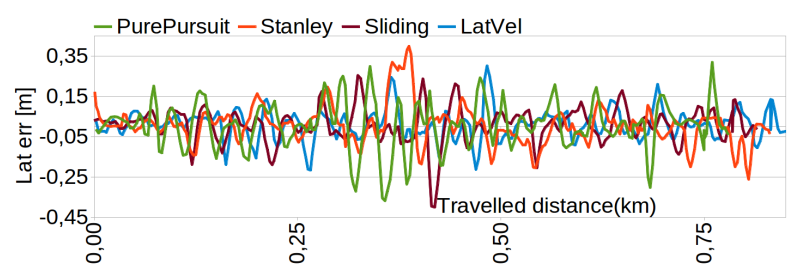

Fig. 9. Lateral error of the four lateral controllers in the first experiment with the real car.

of the ground and some modeling imprecision. However, both cases show the same relative performance between controllers.

For the pure pursuit controller, around $75 \%$ of the error measurements with the real car lie under $11 \mathrm{~cm}$ and the maximum error is around $36 \mathrm{~cm}$. Larger errors occur in tight curves because this controller may cut across a curve if the look ahead distance is not perfectly set for the local curvature of the path.

Quick steering motions create a feeling of discomfort and insecurity for the passenger, especially at high speed. With the pure pursuit controller, the steering control gets smoother as the speed increases, which is pleasant to the passengers.

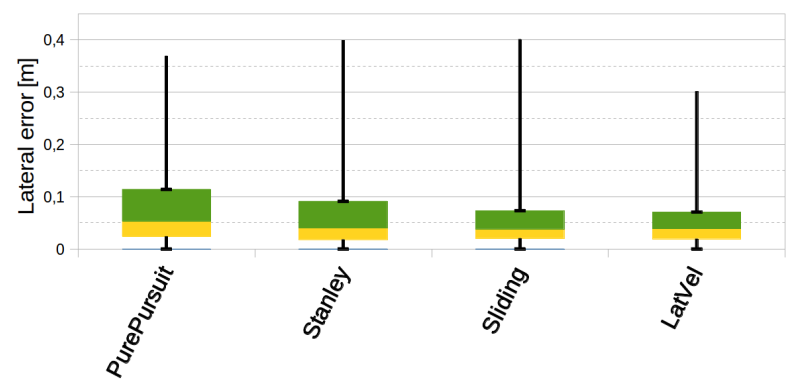

Fig. 10. Lateral error [meters] obtained in experiment 1 with the real car

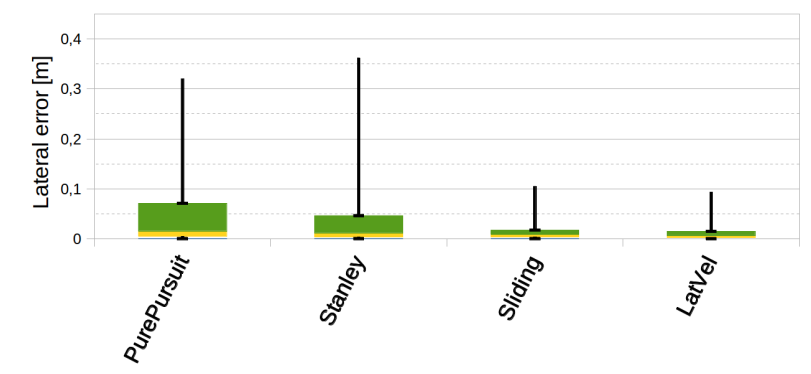

Fig. 11. Lateral error [meters] obtained in experiment 1 with the simulator

The results obtained with the Stanley controller are slightly better than with the pure pursuit in terms of precision: with the real car, $75 \%$ of error measurements lie under $9 \mathrm{~cm}$ and the maximum error is $40 \mathrm{~cm}$. Note that, for the Stanley controller the lateral error is measured as the distance between the center of the front axle to the closest path point (see figure 3), but in figures 9, 1011 and 13 the lateral deviation of the rear axle is represented, to allow comparison. For the Stanley controller, the steering motions are quick and may be a little scary in some parts of the path, thus producing moments of discomfort.

In the case of the kinematic sliding mode controller, the average lateral error is close to that of the lateral velocity controller in both simulation and real case. $75 \%$ of the measurements were under $7 \mathrm{~cm}$ and the maximum value of the error was about $40 \mathrm{~cm}$. Regarding comfort, it is quite acceptable as the movements are smooth.

Finally, with the real car, the proposed lateral error speed controller yielded slightly better results in terms of precision than the sliding mode controller, being the better scorer, with $75 \%$ of the measurements under $6.5 \mathrm{~cm}$ and a maximum error of $30 \mathrm{~cm}$. Regarding passenger's comfort the movements were smooth at all times as the controller is designed to control the lateral speed, avoiding quick lateral movements and producing a feeling of safety. It is also precise enough to track the path without getting too close to road borders. The conclusion is that, at this range of speeds, the lateral error speed controller meets the desired specifications for autonomous navigation.

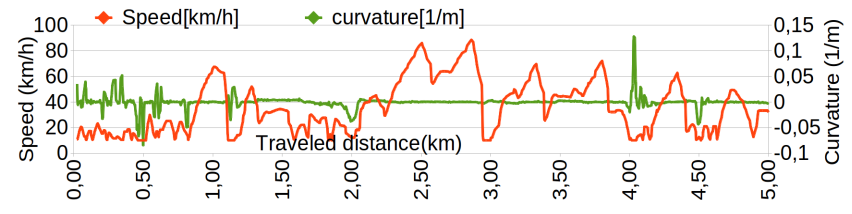

Fig. 12. Profile of the speed (in green) and curvature (in orange) vs distance travelled in $\mathrm{km}$ of experiment 2.

Figure 12 presents the speed and curvature profiles of the path of the second experiment. In this experiment, the four controllers were tested in simulation, limiting the speed to $90 \mathrm{~km} / \mathrm{h}$.

The results obtained are shown in figure 13. The Stanley method showed signs of instability above $25 \mathrm{~km} / \mathrm{h}$ with the parameters used in experiment 1 , so it was decided to use two sets of parameters for speeds below and above $25 \mathrm{~km} / \mathrm{h}$, respectively. The other three controllers showed good stability even for speeds up to $90 \mathrm{~km} / \mathrm{h}$. Moreover, they showed similar performance than in experiment 1 along the whole path. Among the four controllers, the ones that performed best were the kinematic-based controllers, with a maximum error below $10 \mathrm{~cm}$, while the pure pursuit gave a maximum error of $32 \mathrm{~cm}$, with $75 \%$ of the measurements under $5 \mathrm{~cm}$. On the other hand, in spite of using different parameters at high speed, the Stanley controller performed worse than the three other methods at higher speeds, with $75 \%$ of the measurements under $11 \mathrm{~cm}$ and a maximum error of $33 \mathrm{~cm}$.

\section{CONCLUSIONS}

This paper compares three classical path tracking lateral controllers and presents a fourth one, a kinematic controller based on the control of the lateral error velocity. The new controller aims at making lateral movements smoother in order to improve comfort. Two of the other controllers are 


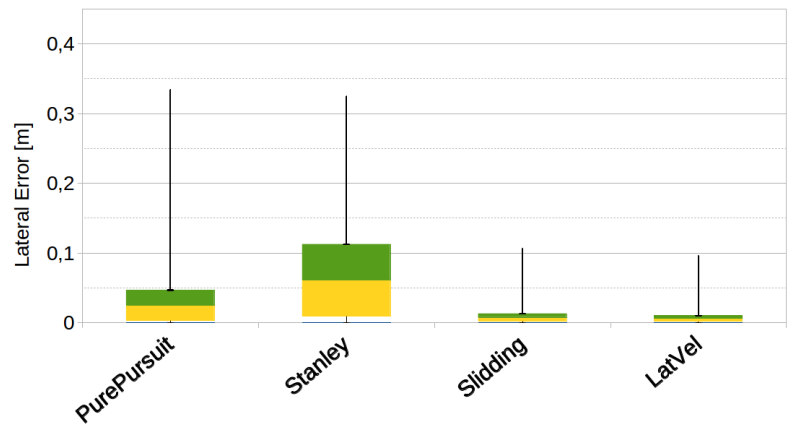

Fig. 13. Distribution of the lateral errors [meters] for experiment 2.

TABLE I

LATERAL CONTROLLERS COMPARISON TABLE

\begin{tabular}{l|l|l|l} 
& Precision & Stability & Smoothness \\
\hline P. Pursuit & Acceptable & Very good & High \\
\hline Stanley & Good at low speed & Bad at higher speeds & Low \\
\hline Sliding & Very Good & Very good & Acceptable \\
\hline LatVel & Very Good & Very good & Good
\end{tabular}

geometric controllers: Pure pursuit and Stanley, and one is a simplified kinematic sliding mode controller. All controllers have been evaluated with a real car and with its modelled version on a simulator. The paths used in the experiments have been pre-recorded in a real environment using a multimap SLAM system [8]. The localization and control system used in simulation is exactly the same as with the real car, the only difference being that in simulation a simplified dynamic model of the car has been used. The relative performance of the controllers is similar in simulation and with the real car at low speeds (up to $30 \mathrm{~km} / \mathrm{h}$ ) but the absolute results are better in simulation. This was expected, since the simulations do not take into account phenomena such as variable friction between the wheels and the road surface, road inclination, etc. Regarding precision at lower speeds, both with the real car and with the simulator, the most accurate controller has proved to be the proposed lateral velocity kinematic controller, while the sliding kinematic controller has shown similar performance. The pure pursuit revealed to be accurate enough for autonomous navigation. Regarding stability, all controllers except the Stanley have shown good stability at all speeds used in the test paths. Regarding passenger comfort and feeling of safety, the best ones were the kinematic controllers and pure pursuit, as the movements are precise enough and very smooth, giving the impression of stability, while the Stanley was precise enough for navigation at low speeds but with quick movements that made the passengers feel unsafe. As shown in the results, the proposed lateral speed controller fits the requirements for road navigation, in terms of precision, smoothness and stability.

\section{ACKNOWLEDGEMENT}

This work was sponsored by the French government research program "Investissements d'avenir" through the RobotEx Equipment of Excellence (ANR-10-EQPX-44).

\section{REFERENCES}

[1] Ali, A.; Garcia, G.; Martinet, P., "Urban platooning using a flatbed tow truck model", in Intelligent Vehicles Symposium, pp. 374-379, Seoul South Korea, June 28 -July 1, 2015

[2] Bertozzi, M.; Broggi, A.; Fascioli, A., "Vision-based intelligent vehicles: State of the art and perspectives", Robotics and Autonomous Systems, Vol. 32, Issue 1, pp. 1-16, 31 July 2000

[3] Canudas de Wit, C.; Siciliano, B.; Bastin, G., "Theory of Robot Control, Series: Communications and Control Engineering", springer, ISBN 3540760547, 1996

[4] Canale, M.; Fagiano, L.; Ferrara, A.; Vecchio, C., "Vehicle Yaw Control via Second-Order Sliding-Mode Technique", IEEE Transactions on Industrial Electronics, vol.55, no.11, pp. 3908-3916, Nov. 2008

[5] Chaib, S.; Netto, M.S.; Mammar, S., "H $\infty$, adaptive, PID and fuzzy control: a comparison of controllers for vehicle lane keeping", in Intelligent Vehicles Symposium, pp. 139-144, 14-17 June 2004

[6] Cheng, D.; Isidori, A.; Respondek, W.; Tarn, T. J., "Exact linearization of nonlinear systems with outputs", Mathematical systems theory, Vol. 21, no 1 , pp. 63-83, 1989

[7] Daviet, P.; Parent, M., "Longitudinal and lateral servoing of vehicles in a platoon", in Intelligent Vehicles Symposium, Tokyo, Japan, pp. 4146, 19-20 September 1996

[8] Dominguez, S.; Khomutenko, B.; Garcia, G.; and Martinet, P., "An optimization technique for positioning multiple maps for self-driving car's autonomous navigation", International Conference on Intelligent Transportation Systems, Gran Canaria, Spain, September 15-18, 2015

[9] Lenain, R.; Thuilot, B.; Cariou, C.; Martinet, P., "High accuracy path tracking for vehicles in presence of sliding: Application to farm vehicle automatic guidance for agricultural tasks", in Autonomous Robots, Kluwer Academic Publishers, vol. 21, n. 1, pp. 79-97, august, 2006

[10] Lenain, R.; Thuilot, B.; Oliver H.; Martinet, P., "High-speed mobile robot control in off-road conditions: A multi-model based adaptive approach", International Conference in Robotics and Automation, Shanghai, China, pp. 6143-6149, 9-13 May 2011

[11] Mammar, S.; Baghdassarian, V.B.; Nouveliere, L., "Speed scheduled vehicle lateral control", International Conference in Intelligent Transportation Systems, pp. 80-85, 1999

[12] Rajamani, R., Tan, H.S.; Law, B.K.; Zhang, W. B., "Demonstration of integrated longitudinal and lateral control for the operation of automated vehicles in platoons", IEEE Transactions on Control Systems Technology, vol.8, no.4, pp. 695-708, Jul 2000

[13] Rajamani, R., "Vehicle dynamics and control", Springer science, ISBN 0387263969, 2006

[14] Sheikholeslam S., Desoer C.A., "Combined Longitudinal and Lateral Control of a Platoon of Vehicles", American Control Conference, 1992. pp. 1763-1767, 24-26 June. 1992.

[15] Shladover, S.E.; Desoer, C.A.; Hedrick, J.K.; Tomizuka, M.; Walrand, J.; Zhang, W.B.; McMahon, D.H.; Peng, H.; Sheikholeslam, S. and McKeown, N., "Automated vehicle control developments in the PATH program", IEEE Transactions Vehicular Technololy, vol. 40, no. 1, pp. 114-130, Feb. 1991

[16] Snider, J. M., "Automatic Steering Methods for Autonomous Automobile Path Tracking", tech. report CMU-RI-TR-09-08, Robotics Institute, Carnegie Mellon University, February, 2009

[17] Tagne, G.; Talj, R.; Charara, A., "Higher-order sliding mode control for lateral dynamics of autonomous vehicles, with experimental validation", in Intelligent Vehicles Symposium, Gold Coast, Australia, pp.678-683, 23-26 June 2013

[18] Thomas, H.; Tomizuka, M., "Fuzzy logic control for lateral vehicle guidance", in Control Systems, vol.14, no.4, pp.55-63, Aug. 1994

[19] Thrun, S.; et al., "Stanley: The robot that won the DARPA grand challenge", Journal of Field Robotics, 23(9):661-692, 2006.

[20] Thuilot, B.; Bom, J.; Marmoiton, F.; and Martinet, P., "Accurate automatic guidance of an urban electric vehicle relying on a kinematic GPS sensor", in the fifth IFAC Symposium on Intelligent Autonomous Vehicles, Lisbon, Portugal, July 5-7th, 2004

[21] Zhao, P.; Chen, J.; Song, Y.; Tao, X.; Xu, T.; Mei, T., "Design of a Control System for an Autonomous Vehicle Based on Adaptive-PID". Int. Journal of Advanced Robotics Systems, Vol. 9, pp.8-18., 2012 